\title{
Neonatal early onset sepsis due to Moraxella osloensis: Case-report and revision of the literature
}

BY EMILIA PARODI, CHIARA GALLETTO, TIZIANA VINCIGUERRA, PAOLA STROPPIANA, INES CASONATO, MARIO FRIGERIO

\section{Abstract}

We report the first case of early-onset systemic neonatal infection associated with Moraxella osloensis bacteriemia in a full term baby. The genus Moraxella is constituted by a group of pleomorphic bacteria obligate aerobes, Gram-negative, oxidase positive and indole negative infrequently isolated from clinical specimens. The organism is rarely reported in the literature as the causative agent of infection in humans, mostly in immunocompromised patients. Only 12 cases of M. osloensisrelated infections during childhood have been reported in the literature so far. This unique report of $\mathrm{M}$. osloensis infection, during the neonatal period, concerns the isolation of the bacteria in purulent secretions from the eyes of a 3-week-old baby with opthalmia. In our patient, the precocity of the onset of symptoms allows us to hypothesize a vertical transmission of the bacteria.

Key words: Moraxella, newborn, sepsis 


\section{Introduction}

The genus Moraxella is constituted by a group of pleomorphic bacteria obligate aerobes, Gram-negative, oxidase positive and indole negative, infrequently isolated from clinical specimens: the main species (M. catarrhalis, M. nonliquefaciens, M. and M. lincolnii osloensis) colonize as saprophytes the upper respiratory tract and occasionally the skin and urogenital tract in humans. (1-3) The clinical significance of M.osloensis isolates may be difficult to determine, because the organism is rarely reported in the literature as the causative agent of infection in humans, mostly immunocompromised patients. (4) We report the first case of early-onset systemic neonatal infection, most likely due to vertical transmission of M. osloensis.

\section{Case report}

The patient is a Caucasian male born vaginally at 39 weeks gestational age. The mother had no history of premature rupture of membranes. Maternal swabs of the lower vagina and rectum had not been performed during pregnancy; incomplete intrapartum prophylaxis against group B streptococcus infection was administered (Ampicillin $2 \mathrm{~g}$ intravenously, only the first dose). There was no other obstetric or family history of interest except for a 3-year-old brother who had received empirical intravenous antibiotic treatment in the first few days of life (no clinical data available).

The neonate's weight, length, and cranial circumference at birth were $4330 \mathrm{~g}$ (LGA, large for gestational age), $53.6 \mathrm{~cm}$ (LGA) and $34.8 \mathrm{~cm}$ (AGA, adequate for gestational age). APGARs were 9 and1o, at 1 and 5 minutes, respectively. At the first neonatal physical examination, no congenital malformations were detected.

One hour after birth the patient developed bradycardia (frequency rate $90 \mathrm{bpm}$ ) and respiratory distress requiring oxygen therapy; lung and cardiac auscultation were normal; no significant findings of other organs were detected on examination. 
Laboratory workup revealed an increased C-reactive protein (0.032 g/l; reference values $<0.005 \mathrm{~g} / \mathrm{l}$ ); full blood count showed a normal white blood cell count and normal values for hemoglobin and platelets; urinalysis was negative.

Following blood culture, on a single sample (with enriched broth), the patient was given empiric intravenous antibiotic treatment with ampicillin+sulbactam. On the second day, blood tests revealed a further rise of C-reactive protein $(0.05 \mathrm{~g} / \mathrm{l})$.

During the following days, blood biochemistry showed a progressive reduction of inflammatory markers and a gradual improvement of the baby's clinical condition was observed.

The newborn was discharged from hospital in good clinical condition ten days later, after completing antibiotic treatment.

On the fifth day after collection, the blood culture came back positive; the day after, subculture plates (blood agar and chocolate agar) showed a Gram negative coccobacillus usually not found on the plate for Gramnegative bacteria (MacConkey agar). At first biochemical identification (instrumental Phoenix Becton Dickinson and manual Biomerieux API $\mathrm{NH}$ ), a bacteria belonging to the genus Moraxella was isolated. To obtain a definitive identification of the species of the organism, 16S rRNA gene sequencing was performed and the blood isolate in the present case was confirmed to be M. osloensis. Our patient's M. osloensis isolate was susceptible to ampicillin+sulbactam.

\section{Discussion}

Moraxella osloensis is rarely reported in the literature as the causative agent of infection in humans. The current medical literature on Moraxella osloensis essentially comes from case reports of infections in humans (osteomyelitis, meningitis, pneumonia, bacteriemia), in immunocompromised patients with malignancies. (4-7)

Regarding isolation of the bacteria in the pediatric population, only 12 cases of M. osloensis-related infections in patients aged o to 15 years have 
been reported in the literature so far. (7-10) Among them, underlying conditions (complement C8 $\beta$ deficiency and cerebrospinal fluid shunt) were present only in two children who both developed meningitis; in the ten remaining pediatric patients the medical history was unremarkable. The most recent cases of M. osloensis concerned a 6- year-old Indian boy with diarrhea and hemolytic uremic syndrome (8) and a 12-year-old Spanish girl with pyomyositis. (9)

We report the first case of early-onset systemic neonatal infection with M. osloensis in a full term baby. To our best knowledge, no neonatal M. osloensis bacteriemias have been described up until now. This unique report of M. osloensis infection during the neonatal period concerns the isolation of the bacteria from purulent eye secretions in a 3-week-old baby with opthalmia. (10) In our patient, the precocity of the onset of symptoms (only one hour after birth) allows us to hypothesize a vertical transmission of the bacteria.

\section{References}

1. Bovre K, Henriksen SD. A new Moraxella species, Moraxella osloensis, and a revised description of Moraxella nonliquefaciens. Int J Syst Bacteriol 1967;17:127-35.

2. Berger U, Falsen E. Distribution of species of Moraxella and moraxellalike organisms in the nasopharynx of healthy human adults. Med Microbiol Immunol 1976;162:239-49.

3. Graevenitz A. Acinetobacter, Alcaligenes, Moraxella, and other nonfermentative Gram-negative bacteria. In: PR Murray, EJ Baron, MA Pfaller, FC Tenover, RH Yolken, editors. Manual of Clinical Microbiology. 6th ed. American Society for Microbiology. Washington; 2008. p. 52032.

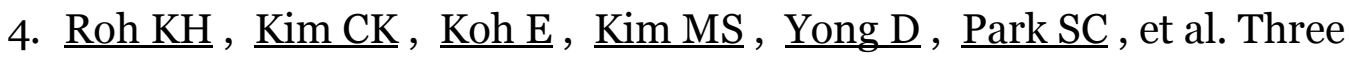
cases of Moraxella osloensis meningitis: a difficult experience in species identification and determination of clinical significance. J Korean Med Sci 2010;25:501-4.

5. Sung JY, Hong SK, Kim EC. The first Korean case of Moraxella osloensis bacteremia in a patient with acute myeloid leukemia. Ann Lab Med 2014;34:256-8. 
6. Vuori-Holopainen E, Salo E, Saxen H, Vaara M , Tarkka E, Peltola H. Clinical "pneumococcal pneumonia" due to Moraxella osloensis: case report and a review. Scand J Infect Dis 2001;33:625-7.

7. Dien Bard J, Lewinski M, Summanen PH, Deville JG. Sepsis with prolonged hypotension due to Moraxella osloensis in a nonimmunocompromised child. J Med Microbiol 2011;60:138-41.

8. Ballal M, Martena S . First case report of Moraxella osloensis diarrhea in a hemolytic uremic syndrome/acute renal failure child from rural coastal India-Manipal, Karnataka. Indian J Pediatr 2013;80:255-7.

9. Bello Gutiérrez P, Cordero Castro C, Alonso Villán E, Carvajal del Castillo O,Carabaño Aguado I, Calatayud Moscos del Prado J. Pyomyositis due to Moraxella osloensis. An Pediatr 2014;80:48-9.

10. Wyplosz B, Mihaila-Amrouche L, Baixench MT, Bigel ML, BerardiGrassias L, Fontaine C, et al. Neonatal Moraxella osloensis Ophthalmia. Emerg Infect Dis 2005;11:1803-4.

Corresponding Author Emilia Parodi

Pediatric and Neonatology Unit AO Ordine Mauriziano I-10128 Turin, Italy Phone: + 390115082428

E-mail: emilia.parodi@unito.it

Article printed from Signa Vitae: http://www.signavitae.com URL to article: http://www.signavitae.com/2015/12/neonatalearly-onset-sepsis-due-to-moraxella-osloensis-case-reportand-revision-of-the-literature/

Copyright (C) 2015 Signa Vitae. All rights reserved. 